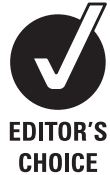

CHOICE
- An additional table is published online only. To view this file please visit the journal online (http://sti.bmj.com/ content/88/3.toc)

${ }^{1}$ Epidemiology and Surveillance Unit, Centre for Infectious Disease Control, National Institute of Public Health and the Environment, Bilthoven, the Netherlands

${ }^{2}$ Department of Infectious Diseases, South Limburg Public Health Service, Geleen, the Netherlands

${ }^{3}$ Division of Infectious Disease Control, Rotterdam Rijnmond Public Health Service Rotterdam, the Netherlands ${ }^{4}$ STI AIDS Netherlands, Amsterdam, the Netherlands ${ }^{5}$ Cluster of Infectious Diseases, Amsterdam Health Service, Amsterdam, the Netherlands

\section{Correspondence to}

Dr Ingrid V F van den Broek Epidemiology and Surveillance Unit, Centre for Infectious Disease Control, National Institute of Public Health and the Environment, P.O. Box 1 (nr 75), BA 3720, the Netherlands; ingrid.van.den.broek@rivm.nl

Accepted 27 November 2011 Published Online First

3 January 2012

\title{
Systematic selection of screening participants by risk score in a chlamydia screening programme is feasible and effective
}

\author{
Ingrid V F van den Broek, ${ }^{1}$ Elfi E H G Brouwers, ${ }^{2}$ Hannelore M Götz, ${ }^{3}$ Jan E A M van Bergen, \\ Eline L M Op de Coul, ${ }^{1}$ Johannes S A Fennema, ${ }^{5}$ Rik H Koekenbier, ${ }^{5}$ Lydia L Pars, ${ }^{4}$ \\ Sander M van Ravesteijn, ${ }^{3}$ Christian J P A Hoebe ${ }^{2}$
}

\section{ABSTRACT \\ Objectives Systematic screening for Chlamydia} trachomatis by individual invitation can be optimised by filtering participants on risk profile, excluding people at no or low risk. The authors investigated this technique in a large-scale chlamydia screening programme in the Netherlands in one rural region where relatively low prevalence was expected $(<2 \%)$.

Methods Invitees were alerted by personal letter to log in to http://www.chlamydiatest.nl and fill in an 8-item questionnaire. Only invitees with sufficient score could proceed to request a test kit. The authors investigated the effect of selection on participation, positivity and acceptability in three screening rounds and on the number needed to invite and the number needed to screen.

Results The selection led to exclusion of $36 \%$ of potential participants and a positivity rate of $4.8 \%$ among participants, achieving similar number needed to screen values in the rural and urban areas. Higher scores were clearly related to higher positivity rates. Persons who were excluded from participation did not have a lower response in the next round. The acceptability study revealed disappointment about exclusion of $30 \%$ of excluded participants but most approved of the screening set-up.

Conclusions Systematic selection of screening participants by risk score is feasible and successful in realising higher positivity rates. A somewhat stricter selection could be applied in the rural and urban areas of the screening programme. Multiple-item selection with a cut-off total score may work better than, more commonly used, selection by single criteria, especially in low-risk populations. Acceptability of selection is high but could still be improved by better communication on expectations

\section{INTRODUCTION}

Chlamydia trachomatis (Ct) infection is the most prevalent treatable sexually transmitted infection (STI). It often affects young heterosexual people, while most other STIs are more common in other risk groups, such as homosexual men. ${ }^{1}{ }^{2}$ Repeated infections occur due to no or limited development of immunity. ${ }^{3}$ Cases often stay asymptomatic, while in the long term, infections can cause serious adverse events, such as pelvic inflammatory disease, tubal pathology and infertility. ${ }^{4}$ To detect asymptomatic cases and prevent these adverse events, screening is the intervention of choice, although good evidence to support the effectiveness of screening is still lacking. ${ }^{5}$

Systematic screening by inviting the whole target population ensures that everyone is reached but has the disadvantage that also people at no/low risk for $\mathrm{Ct}$ will be tested, which might make the screening programme less cost-effective than screening designed to attract people with actual risk behaviour. Selective systematic screening can overcome this issue. A self-selection tool for potential participants in screening has not been applied before in a systematic $\mathrm{Ct}$ screening programme; the unique set-up with a short scoring questionnaire, as we present here, is especially practical with an internet-based program.

In 2008, a Chlamydia Screening Implementation (CSI) was rolled out in three areas of the Netherlands: two large cities, Amsterdam and Rotterdam, and one rural area in South Limburg. ${ }^{67}$ In the cities, all sexually active persons in the target group (men and women aged 16-29 years) were invited to participate without further selection. In the less urbanised region of South Limburg ('Parkstad'), the expected Ct prevalence was lower than in the big cities, and therefore, in line with earlier recommendations, ${ }^{8}$ a selective systematic screening set-up was implemented using a selection risk score modelled on data from the pilot $\mathrm{Ct}{ }^{9}$

Here, we assess the effect of the risk score selection on participation and positivity in CSI and compare the effectiveness of screening expressed as the 'number needed to invite' (NNI) and the 'number needed to screen' (NNS) to find one Ct-positive case ${ }^{10}{ }^{11}$ in South Limburg (selection score) versus Amsterdam and Rotterdam (universal screening). If selection is effective, it reduces the number of people getting tested, while it does hardly or not affect the number of $\mathrm{Ct}$ cases found Hence, the NNI would remain high, while the NNS is reduced. The impact of further selection on the effectiveness of screening is discussed.

\section{METHODS}

\section{Screening and selection through risk score}

The screening programme and evaluation were approved by the Medical Ethics Committee of the VU medical center in Amsterdam (METC number 2007/239). The target population invited for 
screening consisted of all persons from 16 to 29 years old registered in the municipal register. The screening was implemented in a stepped wedge design, that is, a roll-out of invitations cluster by cluster (town-area or village) covering the target population in the course of 1 year; in South Limburg, one-third of the target population was progressively added into the intervention per year in a risk stratified cluster-randomised order; in Amsterdam and Rotterdam, a control group of onesixth of the population was invited only from the second round onwards. Three risk levels defined stratification of clusters: level of urbanisation (in Limburg) or proportion non-Dutch (cities), proportion of people 16-29 years old and proportion with low income per cluster (for more details, see van den Broek et al ${ }^{7}$ ).

All 16-29-year-old persons registered in selected clusters of 10 participating municipalities in Parkstad, South Limburg, received an invitation letter for the chlamydia screening by post. To participate, they were requested to log in to the website http:// www.chlamydiatest.nl with their personal log in code provided in the letter. Online, they were first presented a questionnaire of eight questions. For each question, they could score points; for some issues, different points were assigned per answer for men and women:

- Age 15-19 years (1 point).

- Place of residence (medium urbanised (2 points) and highly urbanised (3 points).

- Medium/lower level of education (2 points).

- Antillean or Surinamese ethnicity (2 points).

- Recent blood loss after sexual contact (women 1 point); Recent increased urge to urinate (men 2 points).

- No condom used at last sexual intercourse (1 point).

- Number of lifetime sex partners (2-5 men (2 points), women (3 points); 6 or more men (3 points), women (5 points).

- New sexual partner in the last 6 months (1 point).

This prediction rule ${ }^{6}$ was developed in pilot Ct. In CSI, the cut-off point was again carefully considered. In first instance, a cut-off of 6 was chosen with the expectation; based on the prior study that by selection of all sexually active participants with a sum score of 6 or higher, the number to be screened would be reduced to $62 \%$, while $7 \%$ of the Ct cases would then be missed (sensitivity 93\%), assuming a prevalence of $3.5 \%$. In 2008 , anyone with a final score of 6 or more was advised to request a $\mathrm{Ct}$ test kit, while low scorers were unable to proceed to request a test kit at the website; the reason of low risk was explained. In 2009, however, the cut-off score was lowered to 5 because a relatively large group was excluded in the previous round. For the purpose of valid comparison, we present the main data based on a cut-off score of 6 for each round.
In Amsterdam and Rotterdam, no risk score questionnaire was used; screening was intended for all sexually active persons. All 16-29-year-old residents logging on to the website (assumed sexually active) were able to request a test kit. The majority of participants voluntarily answered an online questionnaire.

\section{Analyses}

We calculated the proportions excluded from participation by the selection process in South Limburg, the participation among those qualifying to participate and the positivity rate in this group. The relation between individual selection score items and participation and positivity was investigated by scoring profiles and logistic regression. We compared NNI (number invited/number positive) and NNS (number participated/number positive) between the three regions in CSI and in subgroups (gender, age groups and community risk level) with multinomial regression analysis. To estimate an optimal cut-off score for Limburg, we also calculated NNI and NNS and percentage of Ct-positives missed when a higher risk score would have been applied to select participants. We used PASW Statistics (SPSS) V.18 (IBM Corporation).

A small survey $(n=200)$ was held among people who were excluded from participation to assess to what extent the fact that people were excluded from the screening caused problems or negative opinions on the screening and their willingness to be tested in the future (see also Op de Coul et $a l^{12}$ ).

\section{RESULTS}

\section{Selection, participation and positivity rates}

The initial response rate in Limburg, that is, the percentage of persons who filled-in the online questionnaire was $22 \%$ in 2008 , decreasing to $15 \%$ in 2009 and $11 \%$ in round 3 (tables 1 and 2).

The number of points scored in the risk selection questionnaire varied from 0 to 14 ; most persons scored $6-8$ points (figure 1A). Women scored on average higher than men (6.6 with $95 \%$ CI 5.7 to 5.9 vs 5.7 with $95 \%$ CI 6.5 to 6.7, p $<0.001$ ), partly due to the fact that women could score a maximum of 16 points versus 15 points in men. Overall, scores were significantly lower in round 1 than in rounds 2 and $3(p<0.001$, see mean scores in table 1). Most commonly, points were scored for urban residence, medium/lower education level, not using a condom at last sex contact and number of lifetime sex partners. The most influential determinants for actual participation were the same four as well as physical complaints (blood loss after intercourse or increased urination urge), while positivity related mostly to having started a new relationship in the last 6 months and number of lifetime partners.

Table 1 Participation and selection in three screening rounds in South Limburg

\begin{tabular}{|c|c|c|c|c|}
\hline & Round 1 & Round 2 & Round 3 & Overall \\
\hline Invited & 13269 & 25214 & 38428 & 76911 \\
\hline $\begin{array}{l}\text { Filled in questionnaire } \\
\text { (\% of invited) }\end{array}$ & 2.973 (22.4) (M: 16.5; F: 28.8) & 3825 (15.2) (M: 10.9; F: 19.8) & 4381 (11.4) (M: 7.9; F: 15.2) & 11179 (14.5) (M: 10.4; F: 19.0) \\
\hline Average score $(95 \% \mathrm{CI})^{*}$ & $\begin{array}{l}6.16(6.07 \text { to } 6.25) \\
\text { (M: } 5.6 ; \mathrm{F}: 6.5)\end{array}$ & $\begin{array}{l}6.41(6.33 \text { to } 6.48) \\
\text { (M: } 5.9 ; \mathrm{F}: 6.7)\end{array}$ & $\begin{array}{l}6.30(6.23 \text { to } 6.37) \\
(\mathrm{M}: 5.8 ; \mathrm{F}: 6.6)\end{array}$ & $\begin{array}{l}6.30(6.26 \text { to } 6.35) \\
\text { (M: } 5.8 ; \mathrm{F}: 6.6)\end{array}$ \\
\hline $\begin{array}{l}\text { Sufficient score to request } \\
\text { test package ( } \% \text { of respondents) }\end{array}$ & 1851 (62.3) (M: 52.8; F: 68.1)† & 2480 (64.8) (M: 57.3; F: 69.3) & 2777 (63.4) (M: 56.1; F: 67.4) & 7108 (63.5) (M: 55.6; F: 68.3) \\
\hline $\begin{array}{l}\text { Package requested } \\
\text { (\% of selected) }\end{array}$ & $1782(96.3)$ & $2392(96.5)$ & $2665(96.0)$ & $6839(96.2)$ \\
\hline $\begin{array}{l}\text { Package returned ( } \% \text { of package } \\
\text { requests) ( } \% \text { of invitees) }\end{array}$ & $1429(80.2)(10.8)$ & $1927(80.6)(7.6)$ & $2149(80.6)(5.6)$ & $5505(80.5)(7.2)$ \\
\hline Positive test (\% of tested) & 73 (5.1) (M: 5.4; F: 5.0) & 103 (5.3) (M: 4.3; F: 5.8) & 90 (4.2) (M: 3.1; F: 4.7) & 266 (4.8) (M 4.1; F 5.1) \\
\hline
\end{tabular}

*Average score of all persons who filled in the questionnaire.

†Based on sufficient score of 6 . 


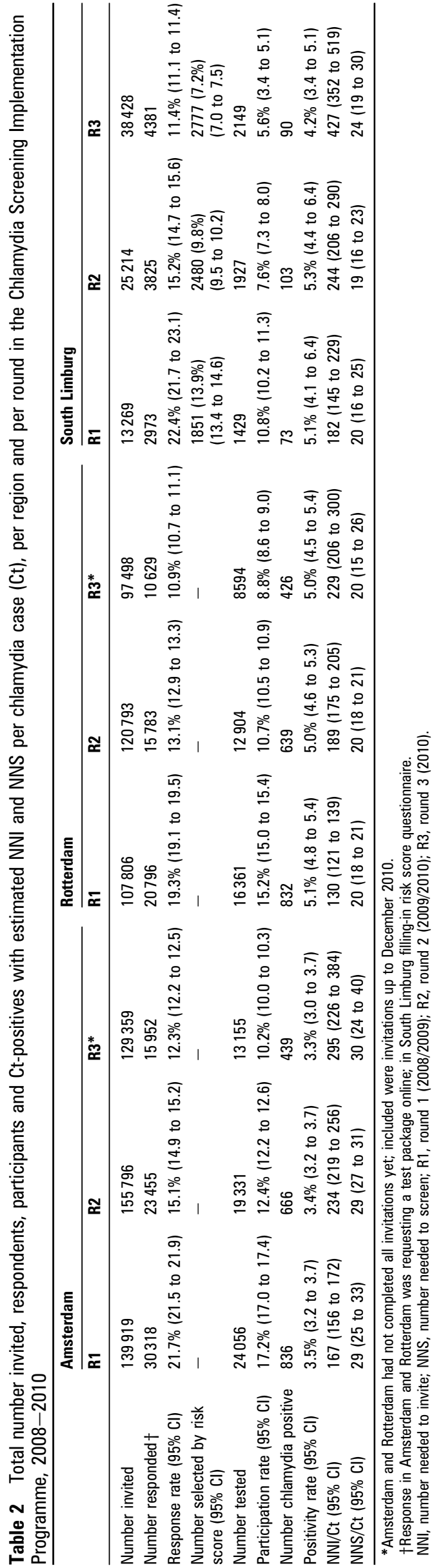

With the overall initial response rate of $14.5 \%$ over three rounds and $63.6 \%$ selection by risk score, $9.2 \%$ of all invitees were actually able to participate. The majority of these $(96 \%)$ requested a test kit, but about one in five $(19.5 \%)$ did not return a sample to the laboratory; an actual 5505 (7.2\% of invitees) participated. The participation rate decreased from $10.8 \%$ to $7.6 \%$ to $5.6 \%$ in the three consecutive rounds. Among the people who were invited in all three rounds, $18 \%$ participated at least once. People who scored too low in a previous round were still as motivated to respond again on a second invitation (36\%) as those who scored high enough (25\%).

Altogether, 266 persons tested positive for $\mathrm{Ct}$. The positivity rate was $5.1 \%$ in the first round, $5.3 \%$ in the second round and $4.2 \%$ in the third round (see tables 1 and 2). As shown in figure $1 \mathrm{~B}$, the positivity rate increased almost linearly with the number of points scored in the risk score questionnaire, both in 2008 and 2009 but not in 2010, when this rise was not seen with scores higher than 9 (numbers are small though: about 200-300 persons scored 10 or more per year).

\section{NNI and NNS}

We calculated that the number of invitations needed to detect one case of chlamydia (NNI) was 182 in the first round in South Limburg and increased to 244 in round 2 and 427 in round 3 . The NNS for one Ct-positive was 20, 19 and 24 in rounds 1, 2 and 3, respectively (see table 2 ).

In comparison, the NNI was significantly lower in Rotterdam than in Amsterdam and in Limburg (table 2), independent of other factors (logistic regression, $\mathrm{p}<0.001$ ). The NNI clearly relates to the higher participation rates in the two cities than in Limburg (table 2, number tested/number invited), where no selection was applied. The NNS was higher in Amsterdam, while values for Rotterdam were quite similar to Limburg. Due to lower participation rates and similar or slightly reduced positivity rates, the NNI increased significantly from round 1 to rounds 2 and 3. Over the three rounds, the NNS remained lower in Rotterdam and Limburg as compared to Amsterdam.

The number of invitations for one positive Ct-case was consistently higher for men than for women (OR 2.4, 95\% CI 2.2 to 2.6 (see online supplementary table 1$)$ ) and varied by age group: the age group 20-24 years had a lower NNI than 16-19years (OR 0.87, 95\% CI 0.81 to 0.94), while the 25-29 years group had a higher NNI (OR 1.41, 95\% CI 1.3 to 1.5). The NNI was higher in low-risk clusters. The NNS for one positive case was also higher for men than for women (OR 1.13, $95 \%$ CI 1.05 to 1.21 ) and increased with age group (OR 1.4 in 20-24 years and 2.5 in 25-29 years compared to $16-19$ years). The NNS was higher in medium- and low-risk areas in Amsterdam and Rotterdam, but this trend was not seen in South Limburg, due to filtering out low-risk participants.

\section{South Limburg: effect of applying stricter selection on NNI and NNS}

To investigate the effect of a stricter selection by applying a higher cut-off point, we compared the NNI and NNS at a hypothetical selection score of $5+$ to $10+$ in rounds 1,2 and 3 . With stricter selection, the NNS would decrease gradually and the NNI would increase (figure 2A), while at the same time, an increasing proportion of the $\mathrm{Ct}$-positives would be missed (figure 2B). At a cut-off point above 7 or 8 , the NNI starts to increase sharply, especially with low participation rates as in round 3. At given participation rates, selection of participants at a cut-off score of 7 points instead of 5 or 6 points would not increase the number of invitations needed per case substantially, 
Figure 1 Number of participants (A) and percentage testing positive for chlamydia (B) by risk score in Chlamydia Screening Implementation, South Limburg, 2008-2010.

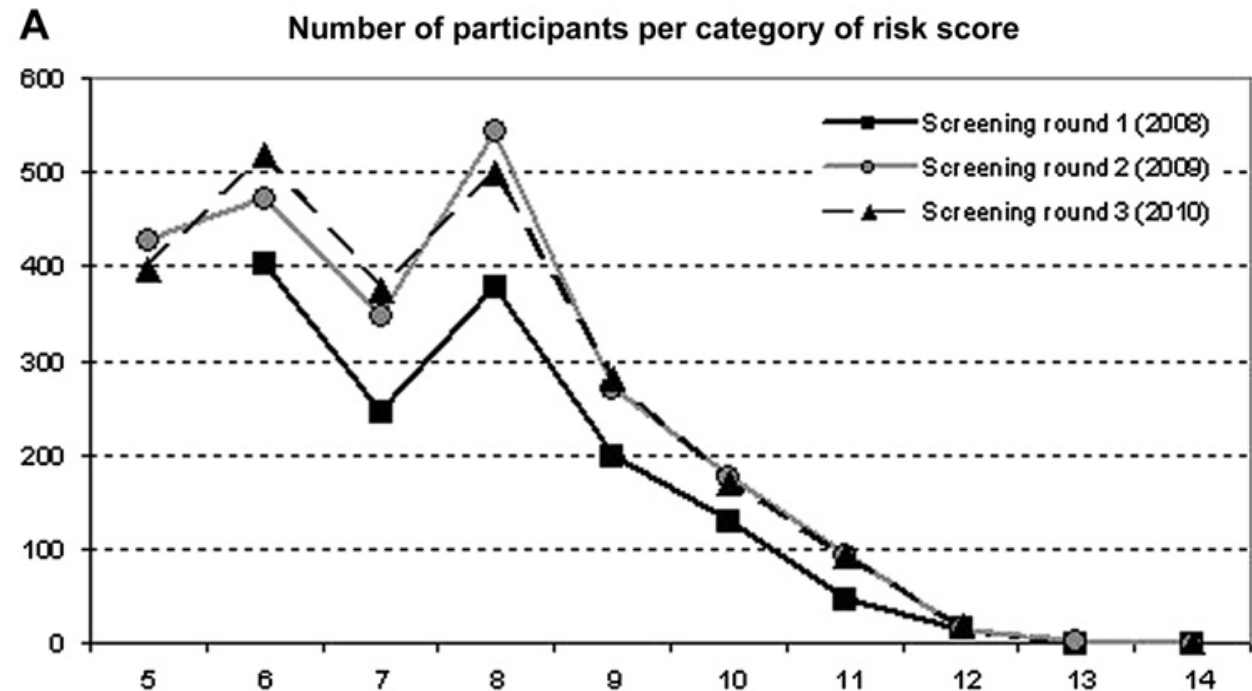

B

Positivity rate by risk score

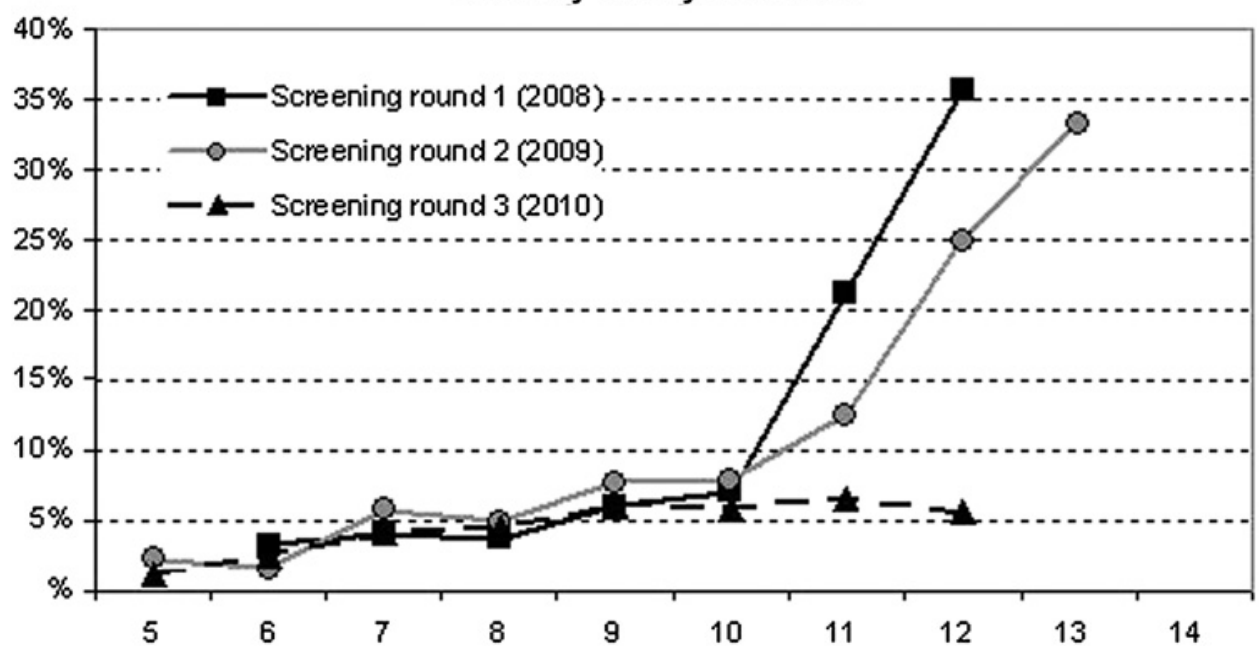

while it would reduce the NNS by about $25 \%$. However, with this stricter selection, $15 \%-20 \%$ of Ct-positives would be missed.

\section{Acceptability of the risk score selection among non-selected} Seventy-six of 200 persons who were excluded from participation $(38 \%)$ responded to the questionnaire on risk score selection, 29 men (33\%) and 47 women (42\%). Of these respondents, 11 (15\%) mentioned that they had not yet been sexually active; hence, they were actually not supposed to fill in the risk score questionnaire. The respondents approved the screening set-up in general (eg, the information was clear, and internet participation was appreciated). More than $30 \%$ indicated they had been disappointed not to be able to participate in CSI. Four persons (5\%) thought that they might have been at risk for chlamydia; six people (8\%) decided to visit a general practitioner or a STI centre for a chlamydia test. Fifty-eight per cent mentioned that they would like to be able to participate in a future screening programme, and $72 \%$ would prefer the same procedure.

\section{DISCUSSION}

In this paper, we describe the innovative use of a prediction rule for Ct-infections and show that selection through risk score in South Limburg worked well, as it was able to capture a population for screening in which the chance to detect a chlamydia infection was higher than in the general population. The positivity rates in the screening were $4.2 \%-5.1 \%$ per round in South Limburg, similar to or even higher than that in the cities Rotterdam (5.3\%) and Amsterdam (3.1\%) and also clearly higher than the national prevalence estimate in an earlier pilot $\mathrm{Ct}$, that is, $2.3 \%$ among sexually active $15-29$-yearolds. $^{8}$

In the targeted population of South Limburg, more invitations needed to be sent out to detect one Ct-infection than in the cities, Amsterdam and Rotterdam, where participation rates were higher because no selection other than the criterion 'being sexually active' was applied. Nevertheless, the NNS was comparable between Limburg and Rotterdam and even higher in Amsterdam. Higher positivity rates in urban areas than in rural areas were expected, based on the pilot study, ${ }^{8}$ but differences between the two cities were also seen, probably relating to different population characteristics (more high-risk clusters in Rotterdam than in Amsterdam) and higher annual testing rates in Amsterdam through a long-established STI clinic ( $21 \%$ of young persons in Amsterdam ${ }^{13}$ indicated to have been tested for STI in the previous year vs $12 \%$ in Rotterdam ${ }^{14}$ ). In the third round in Limburg, when the NNI was more than double of that in the first round, the NNS only increased by a fifth, from 20 to 24 ; hence, the selection remained effective to 
Figure 2 Development of number needed to invite (NNI) and number needed to screen (NNS) (A) and proportion of Ct-positives missed (B) with increasing cut-off point for selection in Chlamydia Screening Implementation, South Limburg, 2008-2010.

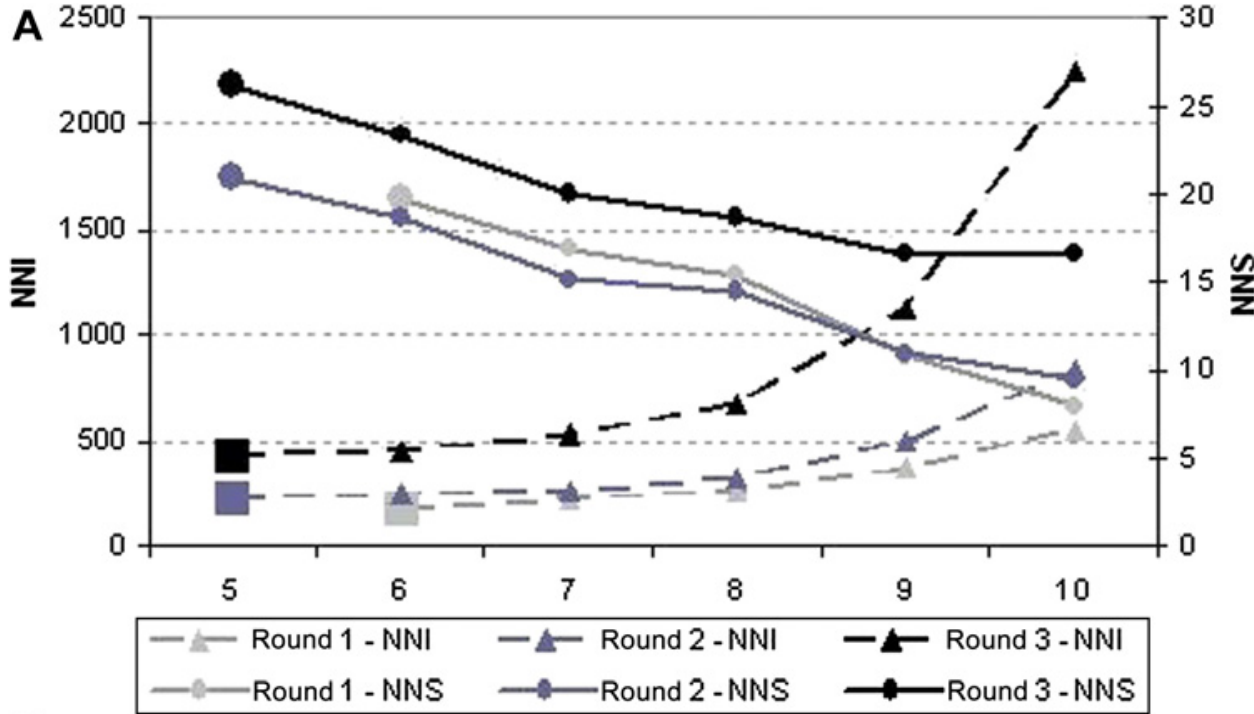

B

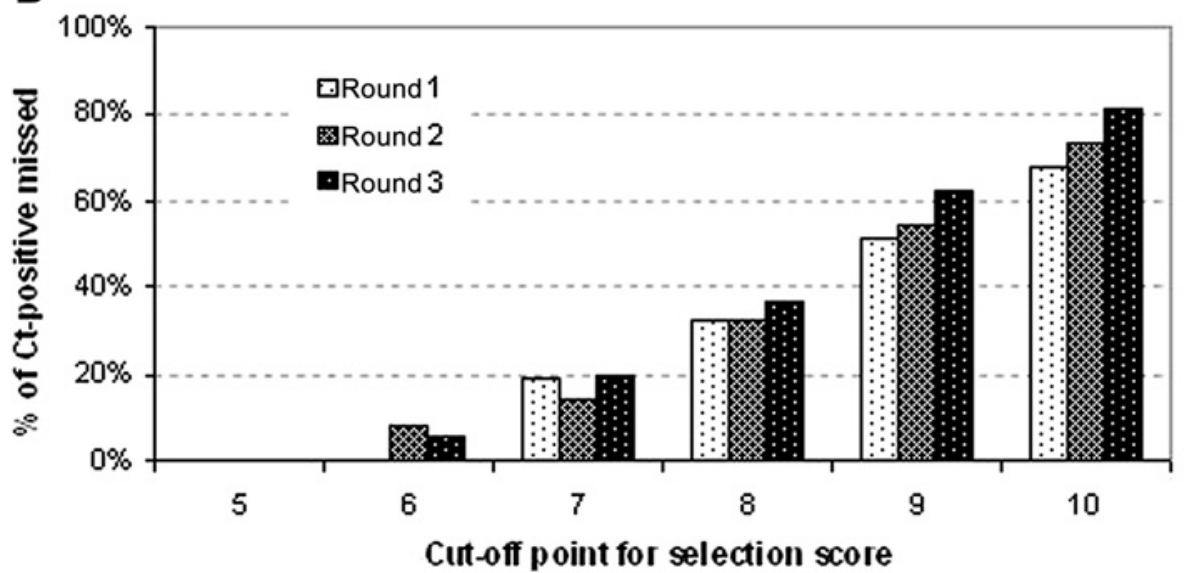

keep the number of tests to be performed low. The findings show that higher scores were clearly related to higher positivity rates. The fact that the highest scores in round 3 were no longer associated with a higher positivity rate may suggest that CSI had some impact, although others show counter effects in highrisk individuals being re-infected repeatedly after screening and treatment. ${ }^{3}$

Selection by risk score can improve cost-effectiveness of a screening programme, provided the costs of testing are a considerable part of the total costs. The extrapolation of the findings in our study shows that a stricter selection could be implemented to make the screening more effective, that is, at a score of 6 or 7 rather than 5 points. However, cut-off scores higher than 7 would result in missing too many Ct-positives. Our results, retrospectively, for the cities suggest that selection by risk score can be applied here as well. Furthermore, our findings indicate that the screening might also become more effective (based on a lower NNI), when rolled out in specific geographic areas, that is, restricted to high- and medium-risk neighbourhoods, or limited to specific groups in the population, that is, inviting only women for screening. Women are more likely to participate in chlamydia screening and have a higher chance to test positive, nevertheless preferably men should be targeted for screening as well. ${ }^{15} 16$

Invitees in South Limburg who were denied the opportunity to be tested because of a low score were frequently disappointed by this. Although they still approved the screening set-up in general, this is a potential drawback of selection and should be addressed by more careful explanation about the selection procedure and the 'momentary' value of the risk score. On the other hand, potential participants in subsequent rounds know about the selection by risk score, so we cannot rule out some misclassification by intention when participants 'lie' to get higher scores to prevent exclusion.

The risk score we used was developed in an earlier pilot study, ${ }^{9}$ where it was calculated that, with a minimum selection score of 6 , the sensitivity was $93 \%$ (proportion of cases detected) by testing $62 \%$ of the population (specificity $38 \%$ ). In our setting, low-risk responders were excluded, so we cannot calculate the sensitivity/specificity, but we tested a similar proportion $(64 \%)$ of the population. The novelty of this scoring system was the use of a point-score per question, different for male and female respondents, adding up to a total score, to which a cut-off point was applied. Other selective screening approaches investigated in the past used single selective criteria and have booked varying results. In a relatively low-risk population, low diagnostic accuracy was reported, ${ }^{17}$ leaving a relatively large part of cases undetected. In more high-risk settings, such as family planning and STI clinics, better results were reported. ${ }^{18} 19$ The criteria used for selection were quite similar, including age, ethnicity, sexual behaviour and symptoms, but the application of a specific score per risk category and a cut-off total score as we used adds to the usefulness of the selection tool in a relatively low-risk population. Within opportunistic 


\section{Key messages}

- Systematic screening for chlamydia will reach all persons in the target group but includes those at no/low risk.

- Selection of participants by risk profile can be attained by a simple short questionnaire linked to a score.

- Selection based on risk score proved highly effective in yielding high positivity rates among the group tested.

- We recommend application of a selection tool in a variety of STI testing settings, especially when combined with online communication.

screening programmes where recruitment is either via the internet, ${ }^{20}$ the general practitioner $^{21}$ or a wider range of healthcare venues, ${ }^{22}$ a risk score selection will be of great value to standardise the focus on higher risk groups, get more insight in who is applying for a test and make people aware of their own risk level.

Internet-based health interventions facilitate interactive communication with the target population; this has been used in a wide range of interventions, from psychological therapy and weight counselling to sexual health information. It enables tailor-made interventions. Self-scoring of risk factors in order to assess one's own risk has been applied widely on medical information websites (ie, breast cancer, diabetes, depression). The use of risk scores directly linked to entry into an intervention programme has been tried before in gastric carcinoma ${ }^{23} 24$ and diabetes ${ }^{25}$; however, it has so far only anecdotally been applied in the context of STI screening. A web-based 'eTriage' tool was used for triage and booking appointments in genitourinary medicine clinics in London. ${ }^{26}$ Risk and symptom selfassessment was used as a way to select male patients visiting a sexual health clinic in the $\mathrm{UK}^{27}$ and computer-assisted selfinterviews for sexual history taking have been shown to be reliable, efficient and highly acceptable in clinical sexual health settings ${ }^{28} 29$ and general practices ${ }^{30}$ in Australia. We think such a selection tool in the setting of an online accessible STI testing website can be very helpful to facilitate case detection and treatment within the target group of young people, who tend to use the internet for private medical matters frequently already.

Investigating the possibilities of a selection by risk score in a larger setting of chlamydia screening, either systematic screening (also in the cities, more national coverage) or more opportunistic (eg, in STI centres before appointments for testing are made), is recommended. For implementation in other countries, the specific questions and answers with scores should be adjusted and validated for the target group and area.

Acknowledgements The authors gratefully acknowledge Dr M.A.B. van der Sande for comments on earlier versions of the manuscript and G. Doornbos and M. Meijer for assistance in data management.

Funding The Dutch Organisation for Health Research and Development (ZonMw). Project number 12.400.0001. They are the financial administrative office for research for the Ministry of Health, Welfare and Sports; the Chlamydia Screening Implementation Programme is being carried out by request of the Ministry of Health, Welfare and Sport.

\section{Competing interests None}

Patient consent The data analysis has been done with completely anonymised data; the results are only presented as aggregated per group. Patient questionnaires were voluntary.
Ethics approval The ethics committee of the Free University of Amsterdam (METC number: 2007/239) has approved the study, which conforms to national and international legislation.

Contributors IVFvdB was the author responsible for design of the study, analysis and writing. EEHGB and CJPAH were involved in the implementation of selective screening in South Limburg and were, together with HMG and ELMOdC, also involved in writing and analysis. JEAMvB and LLP coordinated the Chlamydia Screening Implementation (CSI) Programme. Other coauthors were implementing partners in Rotterdam (HMG and SMvR) and Amsterdam (JSAF and RHK). All authors have commented on draft versions and approved the final version of the manuscript. All authors are part of the CSI Project Group, which is involved in the conception, design and implementation of the CSI Programme. JEAMvB and LLP were responsible for the coordination of the screening in the three regions; CJPAH and EEHGB for the screening in South Limburg, JSAF and RHK in Amsterdam and HMG and SMvR in Rotterdam. IVFvdB drafted the manuscript. Other authors provided feedback for the initial document and contributed to the revision of the paper. All authors read and approved the final draft.

Provenance and peer review Not commissioned; externally peer reviewed.

\section{REFERENCES}

1. European Centre for Disease Prevention and Control. Sexually Transmitted Infections in Europe, 1990-2009. Stockholm: ECDC, 2011

2. Vriend HJ, Koedijk FDH, van den Broek IVF, et al. Sexually Transmitted Infections, Including HIV, in the Netherlands in 2010. RIVM report 210261009. 2011. http:// www.rivm.nl/Thema_s/Infectieziekten/Soa (accessed Oct 2011).

3. Batteiger BE, Xu F, Johnson RE, et al. Protective immunity to Chlamydia trachomatis genital infection: evidence from human studies. J Infect Dis 2010;201 (Suppl 2):S178-89. Review.

4. Geisler WM. Duration of untreated, uncomplicated genital Chlamydia trachomatis infection and factors associated with chlamydia resolution: a review of human studies. J Infect Dis 2010;201(Suppl 2):S104-13.

5. Low N, Bender N, Nartey L, et al. Effectiveness of chlamydia screening: systematic review. Int J Epidemiol 2009;38:435-48.

6. van Bergen JE, Fennema JS, van den Broek IV, et al. Rationale, design, and results of the first screening round of a comprehensive, register-based, Chlamydia screening implementation programme in the Netherlands. BMC Infect Dis 2010;10:293.

7. van den Broek IV, Hoebe CJ, van Bergen JE, et al. Evaluation design of a systematic, selective, internet-based, Chlamydia screening implementation in the Netherlands, 2008-2010: implications of first results for the analysis. BMC Infect Dis 2010; 10:89.

8. van Bergen JE, Götz HM, Richardus JH, et al; PILOT CT study group. Prevalence of urogenital Chlamydia trachomatis increases significantly with level of urbanisation and suggests targeted screening approaches: results from the first national population based study in the Netherlands. Sex Transm Infect 2005;81:17-23.

9. Götz HM, van Bergen JE, Veldhuijzen IK, et al. A prediction rule for selective screening of Chlamydia trachomatis infection. Sex Transm Infect 2005;81:24-30.

10. Nelson HD, Helfand M. Screening for chlamydial infection. Am J Prev Med 2001;20 (Suppl 3):95-107.

11. Rembold CM. Number needed to screen: development of a statistic for disease screening. BMJ 1998;317:307-12. Review.

12. Op de Coul ELM, Weenen TC, van der Sande MAB, et al. Process Evaluation of the Chlamydia Screening Implementation in the Netherlands: Phase 1. RIVM Report 210261006/2009. http://www.rivm.nl/Thema s/Infectieziekten/Soa laccessed May 2011).

13. GGD Amsterdam. Health-Monitor Report 2008. 'Zo gezond is Amsterdam'. 2009. http://www.gezond.amsterdam.n//Beleid-onderzoek-preventie/Gezondheidsmonitors/ Amsterdamse-Gezondheidsmonitor/AGM-2008/Bijlagen-AGM-2008-Eindrapport (accessed Oct 2011).

14. GGD Rotterdam-Rijnmond. Monitor Seksuele Gezondheid. http://www. ggdrotterdamrijnmond.nl/onderzoek/professionals/monitor-seksuele-gezondheid.html (accessed Oct 2011).

15. Low N, Egger M. What should we do about screening for genital chlamydia? Int $J$ Epidemiol 2002;31:891-3.

16. Hart GJ, Duncan B, Fenton KA. Chlamydia screening and sexual health. Sex Transm Infect 2002; 78:396-7.

17. van Valkengoed IG, Morré SA, van den Brule AJ, et al. Low diagnostic accuracy of selective screening criteria for asymptomatic Chlamydia trachomatis infections in the general population. Sex Transm Infect 2000;76:375-80.

18. Marrazzo JM, Fine D, Celum CL, et al. Selective screening for Chlamydial infection in women: a comparison of three sets of criteria. Fam Plann Perspect 1997;29:158-62

19. Marrazzo JM, Celum CL, Hillis SD, et al. Performance and cost-effectiveness of selective screening criteria for chlamydia trachomatis infection in women. Implications for a national chlamydia control strategy. Sex Transm Dis 1997:24:131-41.

20. Gaydos CA, Dwyer K, Barnes M, et al. Internet-based screening for Chlamydia trachomatis to reach non-clinic populations with mailed self-administered vaginal swabs. Sex Transm Dis 2006;33:451-7. Erratum in: Sex Transm Dis 2007; $34: 625$ 
21. Hocking JS, Temple-Smith M, Poznznski S, et al; ACCEPt Consortium. Australian chlamydia control effectiveness pilot: preliminary results from a trial of chlamydia testing in general practice. Poster at the ISSTDR Conference, 10-13 July 2011, Quebec, Canada. Sex Transm Infect 2011:87:A202.

22. NCSP. NCSP Annual Report 2008-2009. 2009. http://www.chlamydiascreening. nhs.uk (accessed Aug 2011).

23. Meining A, Bayerdörffer $E$, Müller $P$, et al. Gastric carcinoma risk index in patients infected with Helicobacter pylori. Virchows Arch 1998;432:311-14.

24. Meining A, Kompisch A, Stolte M. Comparative classification and grading of Helicobacter pylori gastritis in patients with gastric cancer and patients with functional dyspepsia. Scand J Gastroenterol 2003;38: 707-11.

25. Lindström J, Tuomilehto J. The diabetes risk score: a practical tool to predict type 2 diabetes risk. Diabetes Care 2003;26:725-31.
26. Jones R, Menon-Johansson A, Waters AM, et al. eTriage-a novel, web-based triage and booking service: enabling timely access to sexual health clinics. Int J STD AIDS 2010;21:30-3.

27. Pittrof R, McLellan J. Test Not Talk screening for asymptomatic men. Int J STD AIDS 2007; 18:274-5

28. Tideman RL, Chen MY, Pitts MK, et al. A randomised controlled trial comparing computer-assisted with face-to-face sexual history taking in a clinical setting. Sex Transm Infect 2007;83:52-6.

29. Yeung A, Bush M, Cummings $\mathrm{R}$, et al. Use of computerized medical records to determine the feasibility of testing for chlamydia without patients seeing a practitioner. Int J STD AIDS 2010;21:755-7.

30. Bilardi JE, Sanci LA, Fairley CK, et al. The experience of providing young people attending general practice with an online risk assessment tool to assess their own sexual health risk. BMC Infect Dis 2009:9:29.

Letter

Response to 'Comparative performance of culture using swabs transported in Amies medium and the Aptima Combo 2 nucleic acid amplification test in detection of Neisseria gonorrhoeae from genital and extra-genital sites:

\section{a retrospective study'}

The recent article in Sexually Transmitted Infections by Harryman et al assessed the performance of Aptima Combo 2 (AC2) confirmed by Aptima GC (AGC) versus culture and concluded that AC2 with AGC confirmation performed well at genital and extra-genital sites for detection of Neisseria gonorrhoeae (GC). Culture with transport swabs was found to perform poorly for asymptomatic men, symptomatic and asymptomatic women and at extra-genital sites. The authors conclude that consideration should be given on how best to optimise GC culture in settings where direct plating is not feasible.

We strongly agree with them and are pleased to find the accumulating evidence for the performance benefits of AC2 confirmed with AGC. They mentioned that studies by Moss et $a l^{2}$ and Lavelle et $a l^{3}$ concluded that AC2 GC positives were likely to be true positives based on culture and partner data, but point out that both studies confirmed their positives only by repeating the same assay. However, in our later study we confirmed all GC positives by AC2 by retesting residual sample in the AGC assay. ${ }^{4}$

In our study, we looked retrospectively at laboratory dual testing data between August 2006 and April 2008 and reviewed case notes of all patients with a positive result for GC (culture or AC2 confirmed by AGC). Testing was carried out at Macclesfield genitourinary medicine (GUM) clinic (3589 dual nucleic acid amplification test (NAAT) samples from 1930 women and 2470 dual NAAT samples from 1867 men) and in the corresponding community served by the 'Team Chlamydia' office of the National Chlamydia Screening Programme (samples from 1549 men and 7934 women). Of the total 15542 tests performed only one was positive for GC by AC2 but unconfirmed by AGC. There was no culture positive but AC2 negative result in any of our patients tested by both methods. At the GUM clinic, $6(19 \%)$ men and $4(25 \%)$ women would have been undiagnosed if tested only by culture. Of the six men, three were positive at extra-genital sites (pharyngeal swabs) only.

In the community 23 young women would have gone undiagnosed and untreated for GC infection if tested only for chlamydia infection.

The overall positivity for GC in the GUM clinic was $1.3 \%$, the true prevalence being $0.9 \%$ (after excluding already diagnosed cases referred from the community and those presenting as contacts) and that in the community was $0.4 \%$.

Culture alone must now be considered unfit for testing asymptomatic patients and inadequate to meet the challenge of detecting and managing the large number of cases that are to be found outside the GUM clinic setting. ${ }^{5}$ Following the recent guideline $^{6}$ that GC should always be treated with a two antibiotic combination, GC culture may retain its importance for survey and monitoring of changes in antibiotic susceptibility patterns but becomes less essential as a test for every individual patient.

Moncado et $a l^{7}$ evaluated three of the CDC (Centers for Disease Control and Prevention) approaches for confirming GC positive NAAT results and concluded that confirmatory testing was not warranted for genital specimens. With our results and those of Harryman et al and as more evidence accumulates confidence may grow that, at least for $\mathrm{AC} 2$, confirmation is unnecessary.

\section{Mrinalini Mahto, ${ }^{1}$ Harry Mallinson ${ }^{2}$}

${ }^{1}$ East Cheshire Centre for Sexual Health, Macclesfield District General Hospital, East Cheshire NHS Trust,
Macclesfield, UK; ${ }^{2}$ University Hospital Aintree NHS Foundation Trust, Liverpool, UK

Correspondence to Dr Mrinalini Mahto, Department of Sexual Health, Macclesfield District General Hospital, Macclesfield SK10 3BL, UK; mmahto@nhs.net

Competing interests None.

Ethics approval This was a retrospective study and hence ethics approval was not required.

Contributors Both authors contributed equally to writing this letter.

Provenance and peer review Not commissioned; internally peer reviewed.

Accepted 27 November 2011

Published Online First 22 December 2011

Sex Transm Infect 2012;88:211

doi:10.1136/sextrans-2011-050424

\section{REFERENCES}

1. Harryman L, Scofield S, Macleod J, et al. Comparative performance of culture using swabs transported in Amies medium and the Aptima Combo 2 nucleic acid amplification test in detection of Neisseria gonorrhoeae from genital and extra-genital sites: a retrospective study. Sex Transm Infect 2012;88:27-31.

2. Moss S, Mallinson $\mathrm{H}$. The contribution of APTIMA Combo 2 assay to the diagnosis of gonorrhoea in genitourinary medicine setting. Int J STD AIDS 2007:18:551-4.

3. Lavelle S, Jones $\mathrm{KE}$, Mallinson $\mathrm{H}$, et al. Finding, confirming and managing gonorrhoea in a population screened for Chlamydia using the Gen-Probe Aptima Combo 2 assay. Sex Transm Infect 2006;82:221-4.

4. Mahto M, Zia S, Ritchie D, et al. Diagnosis, management and prevalence estimation of gonorrhoea: influences of Aptima Combo 2 assay with alternative target confirmation. Int J STD AIDS 2009:20:315-19.

5. Skidmore S, Copley S, Cordwell D, et al. Positive nucleic acid amplification tests for Neisseria gonorrhoeae in young people tested as part of the National Chlamydia Screening Programme. Int J STD AIDS 2011;22:398-9.

6. Bignell C, FitzGerald M. UK national guideline for the management of gonorrhoea in adults, 2011. Int J STD AIDS 2011:22:541-7.

7. Moncada J, Donegan E, Schachter J. Evaluation of CDC-recommended approaches for confirmatory testing of positive Neisseria gonorrhoeae nucleic acid amplification test results. J Clin Microbiol 2008:46:1614-19. 